Rapid Reviews COVID-19

\title{
Review 3: "Repurposed antiviral drugs for COVID- 19; interim WHO SOLIDARITY trial results"
}

Stephen Shiboski ${ }^{1}$

${ }^{1}$ Professor Emeritus, University of California San Francisco, Epidemiology and Biostatisics, USA

Published on: Nov 19, 2020

DOI: 10.1162/2e3983f5.5bd92277

License: Creative Commons Attribution 4.0 International License (CC-BY 4.0). 


\section{$\underline{\text { RR:C19 Evidence Scale rating by reviewer: }}$}

- Reliable. The main study claims are generally justified by its methods and data. The results and conclusions are likely to be similar to the hypothetical ideal study. There are some minor caveats or limitations, but they would/do not change the major claims of the study. The study provides sufficient strength of evidence on its own that its main claims should be considered actionable, with some room for future revision.

$* * * * * * * * * * * * * * * * * * * * * * * * * * * * * * * * * * * * * * *$

\section{Review:}

\section{General comments}

This manuscript provides a clearly written summary of important results from a wellconducted research study. I have some specific comments focusing on the Methods section, which are relatively minor and could easily be addressed in revision.

\section{Specific comments}

1. Methods, 1st paragraph: The randomization procedure and definition of controls should be explained in more detail. First, if control designation is synonymous with "local standard-of- care," this should be stated and not left as a parenthetical remark. Also, some sense of what constitutes standard of care should also be provided. In particular, is it established that this excluded the study drugs or similar alternatives? What randomization procedures were used? Was any attempt made to balance assignment to the five options across sites? (The Results imply this was not the case, but the procedure should be specified in Methods/Supplementary materials.

2. Statistical analyses, 2nd paragraph, 1st sentence: I suggest replacing "methods plot" with "were used to plot mortality risk through 28 days post-randomization."

3. Statistical analyses, 2nd paragraph, 3rd sentence: This sentence is awkward, partially because of the conditional interpretation implied by starting with "if". Ending with "and assumed to be asymptotically normally distributed" would be clearer. Also, the definition of "expected" isn't clear without further detail (e.g. specifying the null hypothesis). An additional sentence clarifying calculation of expected counts would help. This could also be footnoted in Table 3. Overall, I don't feel the (O-E)/V notation and the inclusion of log-rank statistics in Table 3 add much over the confidence intervals and RR estimates. 
Although not likely to substantively affect reported results, I note that hazard ratios estimated via the log-rank procedure are known to be inconsistent estimators (ref: Lin DY, Dai L, Cheng G, Sailer MO. On confidence intervals for the hazard ratio in randomized clinical trials. Biometrics, 2016, 72(4):1098-1102.) An alternative analysis accounting for this would be preferable. For example, a standard Cox regression analysis adjusting for age (as a continuous or more finely stratified variable) and ventilation status, based on midpoint imputation of interval-censored death times, and accounting for tied event times could have been used (NB: A Cox approach was included in supplementary material).

4. Statistical analyses, 2nd paragraph, 4th sentence: The choice to impute all "uncertain" event times at 7 days is unjustified and the frequency of these is unreported.

5. Statistical analyses, 2nd paragraph, 5th sentence: The frequency of patients censored at day 0 is not reported and needs to be. This sentence could be written more clearly as follows: "Event times for patients with unknown outcomes at the time of analyses were censored at day 0." This implies that these patients were removed from the analysis entirely, and therefore the analysis was technically a "modified intention to treat" approach.

6. Statistical analyses, 2nd paragraph, 6th sentence: How were randomized patients that were discharged treated in the mortality analysis if they were not censored or counted as outcomes? In the prior Endpoints section it is stated: "follow-up ceased at discharge."

7. Statistical analyses, 3rd paragraph, 1st sentence: Denoting the logRR as "b" here serves no purpose in understanding the methods/results.

8. Results, 2nd paragraph, 3rd sentence: The meaning of "Deaths were at median day 8 " is not immediately clear. Something like "Median time to death was 8 (IQR 4-14)" seems clearer to me. 